\title{
Comparison of Chiral Perturbation Theory and QCD Sum Rule Results for Pseudoscalar Isoscalar-Isovector Mixing
}

\author{
Kim Maltman日 \\ Department of Mathematics and Statistics, York University, 4700 Keele St., \\ North York, Ontario, Canada M3J 1P3
}

ADP-95-2/T169

hep-ph/9504252

(June 14, 2018)

\begin{abstract}
The forms of the neutral, non-strange pseudoscalar propagator matrix and mixed axial current correlator, $\left\langle 0\left|T\left(A_{\mu}^{3} A_{\nu}^{8}\right)\right| 0\right\rangle$, are discussed at next-toleading (one-loop) order in chiral perturbation theory, and the results compared to those obtained using QCD sum rules. This comparison provides a check of the truncations employed in the sum rule treatment of the current correlator. Values for the slope of the correlator with $q^{2}$ in the two approaches are found to differ by more than an order of magnitude and the source of this discrepancy is shown to be the incorrect chiral behavior of the sum rule result. 12.39.Fe, 24.85.+p, 21.30.+y, 21.45.+v
\end{abstract}

Typeset using REVTEX 
In the traditional treatment of charge symmetry breaking (CSB) in the meson-exchange framework, contributions to CSB observables arising from isoscalar-isovector meson mixing are obtained under the assumption that the strength of meson mixing is $q^{2}$-independent. A number of recent papers [1] 10], however, have demonstrated that this assumption is suspect. Although the quantity in question (the off-diagonal element of the matrix propagator) is the element of an off-shell Green function and, as such, not in general invariant under allowable field redefinitions (in the sense of Haag's theorem), nonetheless, the existence of $q^{2}$ dependence for any particular choice of interpolating field throws the standard treatment of CSB into question. Of special interest, among the papers mentioned above, is the treatment of $\rho-\omega$ and $\pi-\eta$ mixing using QCD sum rules [7,8] since, without having made any apparent explicit choice for the meson interpolating fields, the authors claim to extract the leading $q^{2}$-dependence of the off-diagonal propagator element. If true, this result would be extremely interesting, suggesting that at least the leading $q^{2}$-dependence, was field reparametrization independent, and hence to be incorporated in all treatments of CSB.

In this paper we critically investigate this claim in the pseudoscalar sector (cf. Ref. [8] ) and come to two main conclusions. The first is that it is not possible to extract the $q^{2}$ dependence of the off-diagonal propagator matrix element from that of the off-diagonal element of the axial vector current correlator matrix. That this is true in general is a consequence of the well-known behavior of quantum field theories under allowed field redefinitions [11] : S-matrix elements are unchanged, but off-shell Green functions are not. The off-diagonal element of the propagator is not a physical observable; the axial vector current correlator is. They cannot, therefore, in general, be related. The second, and more useful, conclusion is that chiral perturbation theory (ChPT) provides formal constraints useful for investigating the reliability of the trunctations employed in the sum rule treatment of the axial vector current correlator. The reason this is true is that ChPT, being constructed solely via symmetry arguments, provides the most general possible representation of the physics of the pseudoscalar Goldstone bosons realizing the exact symmetries of QCD and breaking the approximate chiral symmetries in exactly the way they are broken in QCD. The 
off-diagonal element of the axial current correlator, therefore, has a low-energy expansion in terms of current quark masses, momenta and the low-energy constants of the effective chiral Lagrangian, the terms of which can be calculated in a reliable and systematic manner. The only approximations enter when one truncates this expression to a given order in the chiral expansion. However, even if the expansion converges slowly for a given observable (typically, expansion to one-loop order for $S U(3)_{L} \times S U(3)_{R}$ is sufficient, but a small number of observables are known for which this is not the case - for a general discussion see the recent review by Ecker [12] ) the formal dependence on the current quark masses and momenta obtained to a given order is a rigorous consequence of QCD. For the case at hand, namely the off-diagonal element of the axial current correlator, we will see below that, while the leading chiral behavior of the $q^{2}$-independent part is correctly reproduced by the truncations employed in Ref. [8], the leading chiral behavior of the $q^{2}$-dependent piece is not. The sum rule result for the slope with respect to $q^{2}$ of this correlator, which differs numerically from the one-loop ChPT expression by more than an order of magnitude, thus cannot be correct.

The remainder of the paper is organized as follows. We first revisit the attempt to relate the off-diagonal propagator and axial current correlator matrix elements, fixing our notation in the process. A general expression for the meson pole contributions to the axial current correlator is then given in terms of the meson decay constants and two isospin breaking parameters describing the couplings of the axial currents $A_{\mu}^{3}$ and $A_{\mu}^{8}$ to the physical $\eta$ and $\pi^{0}$, respectively. The calculation of these parameters is then reviewed, the development providing, in addition, an explicit realization of the source of error in the treatment of the relation of the propagator and correlator matrix elements in Ref. [8] . Finally, we compare the one-loop ChPT result for the off-diagonal element of the axial correlator with that obtained via the sum rule analysis. Using the known dependences of the physical meson masses on the current quark masses we show that the truncations employed in the sum rule treatment of Ref. [8] remove the leading chiral behavior of the slope with respect to $q^{2}$, and hence are unsuitable for use in treating this feature of the correlator.

We begin with some notation. Let $\pi, \eta$ represent the physical mixed-isospin $\pi^{o}$ and $\eta$ 
fields, and $\pi_{3}, \pi_{8}$ the pure $I=1,0$ flavor octet neutral fields. Then one has, in general, two mixing angles, $\theta_{\pi}$ and $\theta_{\eta}$, such that, to $\mathcal{O}\left(\theta_{\pi, \eta}\right)$,

$$
\begin{array}{ll}
\pi=\pi_{3}+\theta_{\pi} \pi_{8} & \pi_{3}=\pi-\theta_{\pi} \eta \\
\eta=-\theta_{\eta} \pi_{3}+\pi_{8} & \pi_{8}=\theta_{\eta} \pi+\eta .
\end{array}
$$

(There is, in general, $q^{2}$-dependent mixing so that $\theta_{\pi} \neq \theta_{\eta}$.) To $\mathcal{O}\left(\theta_{\pi, \eta}\right)$, i.e., to first order in isospin breaking, one defines the isospin-breaking parameter, $\theta\left(q^{2}\right)$, by

$$
\Pi_{38}\left(q^{2}\right)=i \int d^{4} x e^{i q \cdot x}\left\langle 0\left|T\left(\pi_{3}(x) \pi_{8}(0)\right)\right| 0\right\rangle \equiv \frac{\theta\left(q^{2}\right)}{\left(q^{2}-m_{\pi}^{2}\right)\left(q^{2}-m_{\eta}^{2}\right)}
$$

where, from Eqns. (1), $\theta\left(q^{2}\right)=q^{2}\left(\theta_{\eta}-\theta_{\pi}\right)+\left(m_{\eta}^{2} \theta_{\pi}-m_{\pi}^{2} \theta_{\eta}\right)$. The axial current correlator, $\Pi_{\mu \nu}^{38}$, is similarly defined via

$$
\Pi_{\mu \nu}^{38}=i \int d^{4} x e^{i q \cdot x}\left\langle 0\left|T\left(A_{\mu}^{3}(x) A_{\nu}^{8}(0)\right)\right| 0\right\rangle \equiv \Pi_{1}^{38}\left(q^{2}\right) q_{\mu} q_{\nu}+\Pi_{2}^{38}\left(q^{2}\right) g_{\mu \nu}
$$

where $A_{\mu}^{3}, A_{\nu}^{8}$ are the 3,8 members of the axial current octet $A_{\mu}^{a}=\bar{q} \gamma_{\mu} \gamma_{5} \frac{\lambda^{a}}{2} q$. In Ref. 8] the authors evaluate this correlator using QCD sum rules, and attempt to determine $\theta\left(q^{2}\right)$ by considering the pseudoscalar pole contributions to the form factor $\Pi_{1}^{38}$. Invoking the PCAC relations $\left\langle 0\left|A_{\mu}^{3}\right| \pi_{3}(q)\right\rangle=i f_{\pi} q_{\mu}$ and $\left\langle 0\left|A_{\mu}^{8}\right| \pi_{8}(q)\right\rangle=i f_{\eta} q_{\mu}$ and, evaluating $\Pi_{1}^{38}$ in the pole approximation, they write

$$
\Pi_{1}^{38}=f_{\pi} f_{\eta}\left[\frac{\theta_{\eta}}{q^{2}-m_{\eta}^{2}}-\frac{\theta_{\pi}}{q^{2}-m_{\pi}^{2}}\right]=\frac{f_{\pi} f_{\eta} \theta\left(q^{2}\right)}{\left(q^{2}-m_{\pi}^{2}\right)\left(q^{2}-m_{\eta}^{2}\right)} .
$$

which would allow the extraction of $\theta\left(q^{2}\right)$ if $\Pi_{1}^{38}$ were known (e. g. from the sum rules treatment). The expression (4), however, is incorrect. The $\pi$ pole term, corresponding to the second term in Eqn. (4), arises from taking

$$
\begin{aligned}
& \left\langle 0\left|A_{\mu}^{3}\right| \pi(q)\right\rangle \equiv\left\langle 0\left|A_{\mu}^{3}\right|\left(\pi_{3}+\theta_{\pi} \pi_{8}\right)(q)\right\rangle=i f_{\pi} q_{\mu} \\
& \left\langle 0\left|A_{\mu}^{8}\right| \pi(q)\right\rangle \equiv\left\langle 0\left|A_{\mu}^{8}\right|\left(\pi_{3}+\theta_{\pi} \pi_{8}\right)(q)\right\rangle=i f_{\eta} \theta_{\pi} q_{\mu}
\end{aligned}
$$

and the first term, corresponding to the $\eta$ pole, from 


$$
\begin{aligned}
& \left\langle 0\left|A_{\mu}^{8}\right| \eta(q)\right\rangle \equiv\left\langle 0\left|A_{\mu}^{8}\right|\left(\pi_{8}-\theta_{\eta} \pi_{3}\right)(q)\right\rangle=i f_{\eta} q_{\mu} \\
& \left\langle 0\left|A_{\mu}^{3}\right| \eta(q)\right\rangle \equiv\left\langle 0\left|A_{\mu}^{3}\right|\left(\pi_{8}-\theta_{\eta} \pi_{3}\right)(q)\right\rangle=-i f_{\pi} \theta_{\eta} q_{\mu}
\end{aligned}
$$

The second relations in Eqns. (5), (6) are, however, false. Although $\left\langle 0\left|A_{\mu}^{3}\right| \pi_{8}\right\rangle=\left\langle 0\left|A_{\mu}^{8}\right| \pi_{3}\right\rangle=$ 0 to leading order in the chiral expansion, beyond leading order, both matrix elements are non-zero. This is inevitable at some order, given that $\mathcal{L}_{Q C D}$ contains a $\Delta I=1$ piece. The result that the mixed-isospin current matrix element vanishes at leading order is a consequence of chiral symmetry, and does not persist beyond this order. Note, moreover, that, at leading order, $\pi_{3}-\pi_{8}$ mixing is $q^{2}$-independent. Thus, at this order, $\theta_{\pi}=\theta_{\eta}$ and the $q^{2}$-dependence of $\theta\left(q^{2}\right)$ vanishes. To determine the $q^{2}$-dependence, one must go beyond leading order, but, beyond leading order, the second of relations (5), (6) are not valid.

The corrected version of the pole approximation to $\Pi_{1}^{38}$ follows from the relations

$$
\begin{array}{ll}
\left\langle 0\left|A_{\mu}^{3}\right| \pi\right\rangle=i f_{\pi} q_{\mu} \quad\left\langle 0\left|A_{\mu}^{8}\right| \pi\right\rangle=i f_{\pi} \epsilon_{1} q_{\mu} \\
\left\langle 0\left|A_{\mu}^{8}\right| \eta\right\rangle=i f_{\eta} q_{\mu} \quad\left\langle 0\left|A_{\mu}^{3}\right| \eta\right\rangle=-i f_{\eta} \epsilon_{2} q_{\mu} .
\end{array}
$$

The isospin-breaking parameters $\epsilon_{1,2}$ have been computed to next-to-leading order in the chiral expansion by Gasser and Leutwyler [13] (the calculation is reviewed below). In Eqn. (7), $f_{\pi}$ is the physical $\pi^{o}$ decay constant (which differs from that for the charged pions only at $\mathcal{O}\left(\left(m_{d}-m_{u}\right)^{2}\right)$ [13], and $f_{\eta}$ the physical $\eta$ decay constant. ChPT implies $f_{\eta} / f_{\pi} \simeq 1.3$ [13] . To this (next-to-leading) order, $\epsilon_{1,2}, f_{\pi}$ and $f_{\eta}$ are all independent of $q^{2}$. From Eqn. (7) one obtains, in the pole approximation,

$$
\Pi_{1}^{38}=\left[\frac{\epsilon_{2} f_{\eta}^{2}}{q^{2}-m_{\eta}^{2}}-\frac{\epsilon_{1} f_{\pi}^{2}}{q^{2}-m_{\pi}^{2}}\right]=\left[\frac{q^{2}\left(\epsilon_{2} f_{\eta}^{2}-\epsilon_{1} f_{\pi}^{2}\right)+\left(m_{\eta}^{2} \epsilon_{1} f_{\pi}^{2}-m_{\pi}^{2} \epsilon_{2} f_{\eta}^{2}\right)}{\left(q^{2}-m_{\eta}^{2}\right)\left(q^{2}-m_{\pi}^{2}\right)}\right] .
$$

At leading order $\epsilon_{1}, \epsilon_{2}, \theta_{\pi}$ and $\theta_{\eta}$ are all equal to $\theta_{0}$, the leading-order $\pi_{3}-\pi_{8}$ mixing angle arising from the leading order off-diagonal term in the meson mass-squared matrix,

$$
\theta_{0}=\frac{\sqrt{3}\left(m_{d}-m_{u}\right)}{4\left(m_{s}-\hat{m}\right)}
$$

where $\hat{m}=\left(m_{u}+m_{d}\right) / 2$. Also at leading order $f_{\pi}=f_{\eta}=F$ so that, at this order, the numerator in Eqn. (8) reduces to $F^{2} \theta_{0}$, which is identical to the leading order expression 
for $f_{\pi} f_{\eta} \theta\left(q^{2}\right)$. The expression (4) is thus correct at leading order in the chiral expansion. Beyond leading order, however, $\epsilon_{2} f_{\eta}^{2} \neq f_{\pi} f_{\eta} \theta_{\eta}$ and $\epsilon_{1} f_{\pi}^{2} \neq f_{\pi} f_{\eta} \theta_{\pi}$ (see below), and knowing the numerator in Eqn. (8) no longer allows one to obtain that of Eqn. (3). In particular, the crucial $q^{2}$-dependence of $\theta\left(q^{2}\right)$, which arises only at next-to-leading order and beyond, is completely inaccessible from the $q^{2}$-dependence of the numerator of (8).

We now provide explicit expressions for all quantities appearing in the above discussion, at one-loop order in the chiral expansion, obtained using the low-energy effective chiral Lagrangian, $\mathcal{L}_{\text {eff }}$, of Ref. [13. All notation is as defined there. We also keep terms only to first order in isospin breaking, i.e., drop terms of $\mathcal{O}\left(\left(m_{d}-m_{u}\right)^{2}\right)$.

Let us first discuss the meson propagator matrix, for the standard choice of meson fields [13. $\pi_{3}^{(0)}, \pi_{8}^{(0)}$ are the bare $\pi_{3}, \pi_{8}$ fields. The bare propagator matrix, to one-loop order, is of the form

$$
\Delta(q)=\left(\begin{array}{cc}
Z_{3}^{-1}\left(q^{2}-m_{3}^{2}\right) & \ell_{38} \\
\ell_{38} & Z_{8}^{-1}\left(q^{2}-m_{8}^{2}\right)
\end{array}\right)^{-1}
$$

where $Z_{3}, Z_{8}$ and $m_{3}^{2}, m_{8}^{2}$ are the $\pi_{3}^{(0)}, \pi_{8}^{(0)}$ one-loop wavefunction renormalization constants and squared-masses in the isospin symmetry limit. Expressions for $m_{3}^{2}$ and $m_{8}^{2}$ in terms of the parameters of $\mathcal{L}_{\text {eff }}$ are given in Ref. [13], and those for $Z_{3}, Z_{8}$ and $\ell_{38}$ are easily computable from $\mathcal{L}_{\text {eff }}$. In what follows we write $Z_{3}^{-1}=1+z_{3}, Z_{8}^{-1}=1+z_{8}$ and $\ell_{38}=\ell_{(0)}+\ell^{(1) 0}+\ell^{(1) 1} q^{2}$. $\ell_{(0)}, \ell^{(1) 0}$ and $\ell^{(1) 1}$ are $q^{2}$-independent and $\mathcal{O}\left(m_{d}-m_{u}\right) . \ell_{(0)}$ is of leading chiral order and $z_{3}, z_{8}, \ell^{(1) 0}, \ell^{(1) 1}$ next-to-leading order. $m_{3}^{2}$ and $m_{8}^{2}$ contain both leading and next-to-leading pieces. The renormalized $\pi_{3}, \pi_{8}$ fields, $\pi_{t}^{r}$ and $\pi_{e}^{r}$, are obtained, as usual, by

$$
\begin{aligned}
& \pi_{t}^{r}=\left(Z^{-1 / 2}\right)_{33} \pi_{3}^{(0)}+\left(Z^{-1 / 2}\right)_{38} \pi_{8}^{(0)} \\
& \pi_{e}^{r}=\left(Z^{-1 / 2}\right)_{83} \pi_{3}^{(0)}+\left(Z^{-1 / 2}\right)_{88} \pi_{8}^{(0)}
\end{aligned}
$$

where $Z$ is the wavefunction renormalization matrix. From Eqn. (10) one has

$$
Z^{-1 / 2}=\left(\begin{array}{cc}
1+\frac{1}{2} z_{3} & \frac{1}{2} \ell^{(1) 1} \\
\frac{1}{2} \ell^{(1) 1} & 1+\frac{1}{2} z_{8}
\end{array}\right)
$$


and from this one obtains the renormalized propagator matrix, to $\mathcal{O}\left(m_{d}-m_{u}\right)$,

$$
\Delta^{(r)}(q)=\left(\begin{array}{cc}
q^{2}-m_{3}^{2} & -m_{t e}^{2} \\
-m_{t e}^{2} & q^{2}-m_{8}^{2}
\end{array}\right)^{-1}
$$

where $m_{t e}^{2}=-\left[\ell_{(0)}+\ell^{(1) 0}+\frac{1}{2} \ell^{(1) 1}\left(m_{3}^{2}+m_{8}^{2}\right)-\frac{1}{2} \ell_{(0)}\left(z_{3}+z_{8}\right)\right]$. Using the explicit expressions for $z_{3}, z_{8}, \ell_{(0)}, \ell^{(1) 0}$ and $\ell^{(1) 1}$, one may verify that $m_{t e}^{2}$ is now finite, and diagonalize the mass-squared matrix to find the physical, renormalized $\pi^{0}, \eta$ fields. Writing

$$
\begin{aligned}
& \pi^{0}=\pi_{t}^{r}+\theta_{r} \pi_{e}^{r} \\
& \eta=-\theta_{r} \pi_{t}^{r}+\pi_{e}^{r}
\end{aligned}
$$

with $\theta_{r}=-m_{t e}^{2} /\left(m_{8}^{2}-m_{3}^{2}\right)$, one finds, explicitly,

$$
\begin{aligned}
\theta_{r}= & \theta_{0}\left[1-3 \mu_{\pi}+2 \mu_{K}+\mu_{\eta}+\left(\frac{3 m_{8}^{2}+m_{3}^{2}}{64 \pi^{2} F^{2}}\right)\left(1+\frac{m_{\pi}^{2}}{\bar{m}_{K}^{2}-m_{\pi}^{2}} \log \left(m_{\pi}^{2} / \bar{m}_{K}^{2}\right)\right)\right. \\
& \left.-\frac{32 B_{0}\left(m_{s}-\hat{m}\right)}{F^{2}}\left(3 L_{7}^{r}+L_{8}^{r}\right)\right] \equiv \theta_{0}\left(1+\delta \theta_{r}\right)
\end{aligned}
$$

where $\mu_{P}=\left[m_{P}^{2} \log \left(m_{P}^{2} / \mu^{2}\right)\right] / 32 \pi^{2} F^{2}$ with $\mu$ the (dimensional regularization) renormalization scale, $L_{k}^{r}$ are the renormalized low-energy constants as defined in Ref. [13], $\bar{m}_{K}^{2}$ is the average $K$ mass-squared and $F, B_{0}$ are the two constants appearing in the lowest order part of the chiral Lagrangian ( $F=f_{\pi}$ in leading order). The expression (14) is not yet of use to us since the $\pi_{t}^{r}$ and $\pi_{e}^{r}$ fields have mixed isospin. We may re-write (14) as

$$
\begin{aligned}
& \pi^{0}=\left(1+\frac{1}{2} z_{3}\right)\left(\pi_{3}^{(0)}+\hat{\theta}_{1} \pi_{8}^{(0)}\right) \\
& \eta=\left(1+\frac{1}{2} z_{8}\right)\left(-\hat{\theta}_{2} \pi_{3}^{(0)}+\pi_{8}^{(0)}\right)
\end{aligned}
$$

where

$$
\begin{aligned}
\hat{\theta}_{1}= & \theta_{0}\left[1-\frac{7}{3} \mu_{\pi}+\frac{4}{3} \mu_{K}+\mu_{\eta}+\left(\frac{3 m_{\eta}^{2}+m_{\pi}^{2}}{64 \pi^{2} F^{2}}\right)\left(1+\frac{m_{\pi}^{2}}{\bar{m}_{K}^{2}-m_{\pi}^{2}} \log \left(m_{\pi}^{2} / \bar{m}_{K}^{2}\right)\right)\right. \\
& \left.+\left(\frac{m_{\eta}^{2}-m_{\pi}^{2}}{64 \pi^{2} F^{2}}\right)\left(1+\log \left(\bar{m}_{K}^{2} / \mu^{2}\right)\right)-\frac{32\left(\bar{m}_{K}^{2}-m_{\pi}^{2}\right)}{F^{2}}\left(3 L_{7}^{r}+L_{8}^{r}\right)\right] \equiv \theta_{0}\left(1+\delta \hat{\theta}_{1}\right) \\
\hat{\theta}_{2}= & \theta_{0}\left[1-\frac{11}{3} \mu_{\pi}+\frac{8}{3} \mu_{K}+\mu_{\eta}+\left(\frac{3 m_{\eta}^{2}+m_{\pi}^{2}}{64 \pi^{2} F^{2}}\right)\left(1+\frac{m_{\pi}^{2}}{\bar{m}_{K}^{2}-m_{\pi}^{2}} \log \left(m_{\pi}^{2} / \bar{m}_{K}^{2}\right)\right)\right. \\
& \left.-\left(\frac{m_{\eta}^{2}-m_{\pi}^{2}}{64 \pi^{2} F^{2}}\right)\left(1+\log \left(\bar{m}_{K}^{2} / \mu^{2}\right)\right)-\frac{32\left(\bar{m}_{K}^{2}-m_{\pi}^{2}\right)}{F^{2}}\left(3 L_{7}^{r}+L_{8}^{r}\right)\right] \equiv \theta_{0}\left(1+\delta \hat{\theta}_{2}\right) .
\end{aligned}
$$


The $\delta \hat{\theta}_{1,2}$ are next-to-leading order quantities. If $\ell_{38}$ had had no $q^{2}$-dependence, we would have had $\hat{\theta}_{1}=\hat{\theta}_{2}$ at this point. To compare with Eqn. (1) we recast the above results in terms of the isospin-pure renormalized fields, $\pi_{3}^{(0) r}$ and $\pi_{8}^{(0) r}$, i.e., the renormalized fields in the isospin symmetry limit, $\pi_{3}^{(0) r} \equiv\left(1+\frac{1}{2} z_{3}\right) \pi_{3}^{(0)}$ and $\pi_{8}^{(0) r} \equiv\left(1+\frac{1}{2} z_{8}\right) \pi_{8}^{(0)}$, obtaining

$$
\begin{aligned}
& \pi^{0}=\pi_{3}^{(0) r}+\theta_{0}\left(1+\delta \hat{\theta}_{1}+\frac{1}{2} z_{3}-\frac{1}{2} z_{8}\right) \pi_{8}^{(0) r} \\
& \eta=-\theta_{0}\left(1+\delta \hat{\theta}_{2}+\frac{1}{2} z_{8}-\frac{1}{2} z_{3}\right) \pi_{3}^{(0) r}+\pi_{8}^{(0) r}
\end{aligned}
$$

Thus, formally, we find $\theta_{\pi}=\theta_{0}\left(1+\delta \hat{\theta}_{1}+\frac{1}{2} z_{3}-\frac{1}{2} z_{8}\right), \theta_{\eta}=\theta_{0}\left(1+\delta \hat{\theta}_{2}+\frac{1}{2} z_{8}-\frac{1}{2} z_{3}\right)$.

Let us turn to the axial current correlator, $\Pi_{\mu \nu}^{38}$. Although the low-energy representation of the product $A_{\mu}^{3} A_{\nu}^{8}$ contains contact terms, these do not affect $\Pi_{1}^{38}$ to next-to-leading order, so we may restrict our attention to the product of representations of the individual currents. These are easily read off from $\mathcal{L}_{\text {eff }}$ of Ref. [13], with the result

$$
\begin{aligned}
A_{\mu}^{3}= & -F \partial_{\mu} \pi_{3}^{(0)}-\frac{16 B_{0}}{F}\left(m_{s}+2 \hat{m}\right) L_{4} \partial_{\mu} \pi_{3}^{(0)}-\frac{16 B_{0}}{F} \hat{m} L_{5} \partial_{\mu} \pi_{3}^{(0)} \\
& +\frac{1}{3 F}\left(4 \pi^{+} \pi^{-}+K^{+} K^{-}+K^{0} \bar{K}^{0}\right) \partial_{\mu} \pi_{3}^{(0)} \\
& +\frac{8 B_{0}\left(m_{d}-m_{u}\right)}{\sqrt{3} F} L_{5} \partial_{\mu} \pi_{8}^{(0)}+\frac{1}{\sqrt{3} F}\left(K^{+} K^{-}-K^{0} \bar{K}^{0}\right) \partial_{\mu} \pi_{8}^{(0)}+\cdots \\
A_{\mu}^{8}= & -F \partial_{\mu} \pi_{8}^{(0)}-\frac{16 B_{0}}{F}\left(m_{s}+2 \hat{m}\right) L_{4} \partial_{\mu} \pi_{8}^{(0)}-\frac{16 B_{0}}{3 F}\left(2 m_{s}+\hat{m}\right) L_{5} \partial_{\mu} \pi_{8}^{(0)} \\
& +\frac{1}{F}\left(K^{+} K^{-}+K^{0} \bar{K}^{0}\right) \partial_{\mu} \pi_{8}^{(0)} \\
& +\frac{8 B_{0}\left(m_{d}-m_{u}\right)}{\sqrt{3} F} L_{5} \partial_{\mu} \pi_{3}^{(0)}+\frac{1}{\sqrt{3} F}\left(K^{+} K^{-}-K^{0} \bar{K}^{0}\right) \partial_{\mu} \pi_{3}^{(0)}+\cdots
\end{aligned}
$$

where the $+\cdots$ indicates terms higher order in the fields, and other terms which do not contribute to $\prod_{1}^{38}$ to one-loop order. As claimed earlier, one sees, beyond the leading order terms $-F \partial_{\mu} \pi_{3}^{(0)}$ in $A_{\mu}^{3}$ and $-F \partial_{\mu} \pi_{8}^{(0)}$ in $A_{\mu}^{8}$, terms which will couple $A_{\mu}^{3}$ to $\pi_{8}$ and $A_{\mu}^{8}$ to $\pi_{3}$. From the expressions (19) for $A_{\mu}^{3,8}$ and the inverted form of the relations (16), i.e.,

$$
\begin{aligned}
& \pi_{3}^{(0)}=\left(1-\frac{1}{2} z_{3}\right) \pi^{0}-\theta_{0}\left(1+\delta \hat{\theta}_{1}-\frac{1}{2} z_{8}\right) \eta \\
& \pi_{8}^{(0)}=\left(1-\frac{1}{2} z_{8}\right) \eta+\theta_{0}\left(1+\delta \hat{\theta}_{2}-\frac{1}{2} z_{3}\right) \pi^{0}
\end{aligned}
$$


(valid to $\mathcal{O}\left(m_{d}-m_{u}\right)$, and to next-to-leading order in the chiral expansion) one may easily verify the results of Gasser and Leutwyler for $f_{\pi}, f_{\eta}, \epsilon_{1}$ and $\epsilon_{2}$. The following equivalent forms display explicitly the problems with the second of Eqns. (5), (6):

$$
\begin{gathered}
\left\langle 0\left|A_{\mu}^{3}\right| \eta(q)\right\rangle \equiv-i f_{\eta} \epsilon_{2} q_{\mu}=-i f_{\pi} q_{\mu}\left(\theta_{\eta}+c_{12}+\frac{8 B_{0}\left(m_{d}-m_{u}\right)}{\sqrt{3} F^{2}} L_{5}+\frac{\left(\hat{L}_{K^{+}}-\hat{L}_{K^{0}}\right)}{\sqrt{3} F^{2}}\right) \\
\left\langle 0\left|A_{\mu}^{8}\right| \pi^{0}(q)\right\rangle \equiv i f_{\pi} \epsilon_{1} q_{\mu}=i f_{\eta} q_{\mu}\left(\theta_{\pi}-c_{12}-\frac{8 B_{0}\left(m_{d}-m_{u}\right)}{\sqrt{3} F^{2}} L_{5}-\frac{\left(\hat{L}_{K^{+}}-\hat{L}_{K^{0}}\right)}{\sqrt{3} F^{2}}\right)
\end{gathered}
$$

where $\hat{L}_{P}$ is the tadpole loop integral $\hat{L}_{P}=\mu^{4-d} \int \frac{d^{d} k}{(2 \pi)^{d}} \frac{i}{\left(k^{2}-m_{P}^{2}\right)}$ and $c_{12}=\theta_{0}\left(\delta \hat{\theta}_{1}-\delta \hat{\theta}_{2}+z_{3}-\right.$ $\left.z_{8}\right)$. The first terms on the right hand sides of Eqns. (21), (22) are the only ones retained in the treatment of Ref. [8] . The remaining terms are present due to the direct couplings of $\pi_{8}^{(0) r}$ (the leading piece of $\eta$ ) to $A_{\mu}^{3}$, at $\mathcal{O}\left(m_{d}-m_{u}\right.$ ), and of $\pi_{3}^{(0) r}$ (the leading piece of $\pi^{0}$ ) to $A_{\mu}^{8}$, at $\mathcal{O}\left(m_{d}-m_{u}\right)$. As already expected on general principles, the second of Eqns. (5), (6) are seen explicitly to be incorrect at next-to-leading order as, in consequence, is Eqn. (4), which is based on them: the isospin-breaking parameter, $\theta\left(q^{2}\right)$, of the pseudoscalar propagator matrix cannot be extracted directly from the mixed axial current correlator.

It is important to stress that this conclusion is inescapable, independent of whether or not the chiral expansions for $\epsilon_{1,2}$ are well-converged at one-loop order. The $q^{2}$-dependences of $\Pi_{1}^{38}$ and $\theta\left(q^{2}\right)$ arise only at one-loop, and not at leading, order in the chiral expansion. Eqns. (21), (22) demonstrate that terms of the same order as those kept must be dropped for the $q^{2}$-dependent piece of the numerator of Eqn. (8) to reduce to $f_{\pi} f_{\eta} \theta\left(q^{2}\right)$. Since such a procedure would be inconsistent (moreover the additional terms are not small, numerically), we confirm that the general argument, based on the behavior of quantum field theories under field redefinitions, is not accidentally evaded in the pseudoscalar sector.

Let us now turn to the more interesting point of using the chiral expansion as a constraint on the truncations employed in the sum rule treatment of the axial current correlator. For this we will give the explicit expressions for $\Pi_{1,2}^{38}$ to one-loop in ChPT and compare the formal quark mass dependences of these expressions with those of the results of the sum rule analysis. The latter may be obtained from the results of Ref. [8] (written in terms of the physical $\pi^{0}, \eta$ masses) by using the known leading- plus next-to-leading expansions for 
$m_{\pi, \eta}$ in terms of the quark masses [13]. It is important to remember that the axial current correlator is a physical object. As such, its dependence on $q^{2}$ is not to be identified with the $q^{2}$-dependence of the angle which diagonalizes the inverse propagator matrix (as obtained in Refs. [2,6] ). That angle, like $\theta\left(q^{2}\right)$ (though distinct from it), is dependent on the choice of meson fields.

The low-energy representation of the product of axial currents, $A_{\mu}^{3} A_{\nu}^{8}$, consists of two parts, (1) the product of the low-energy representations of the individual currents, as given in Eqns. (19), and (2) a set of contact terms associated with those terms in $\mathcal{L}_{\text {eff }}$ quadratic in the external axial fields. To next-to-leading order, explicit calculation shows that the product of the representations of the individual currents contributes only to $\Pi_{1}^{38}$, and the contact terms only to $\Pi_{2}^{38}$. For $\Pi_{2}^{38}$ one finds

$$
\Pi_{2}^{38}=\frac{B_{0}\left(m_{d}-m_{u}\right)}{\sqrt{3}}\left[\frac{3}{32 \pi^{2}}\left(\log \left(\bar{m}_{K}^{2} / \mu^{2}\right)+1\right)-8 L_{5}^{r}\right] .
$$

Although not discussed in detail in Ref. [8] , the pole approximation employed there leads to a $\Pi_{2}^{38}$ (as defined here) which is proportional to $q^{2}$ and hence vanishes as $q^{2} \rightarrow 0$. It follows, from Eqn. (23), that $\Pi_{2}^{38}$ cannot be properly modelled using only pseudovector meson pole terms, as in Ref. [8] . Turning to the remaining form factor, $\Pi_{1}^{38}$, we note that, to this order in the chiral expansion, the pole approximation is exact. The correct one-loop expression for $\Pi_{1}^{38}$ is, therefore, that already written down in Eqn. (8), with $f_{\pi}, f_{\eta}, \epsilon_{1}$ and $\epsilon_{2}$ the oneloop expressions of Ref. [13]. In obtaining numerical results below we have rescaled the values of $\epsilon_{1,2}$ quoted in Ref. [13] by a factor of 1.22 , in order to account for the larger-thanexpected violations of Dashen's theorem [14 [16]. We thus have as input $\epsilon_{1}=1.67 \times 10^{-2}$, $\epsilon_{2}=1.35 \times 10^{-2}, f_{\pi}=93 \mathrm{MeV}$, and $f_{\eta} / f_{\pi}=1.3$. Then, setting $g_{\eta}=f_{\eta}^{2} \epsilon_{2}$ and $g_{\pi}=f_{\pi}^{2} \epsilon_{1}$ and rewriting Eqn. (8) in the form

$$
\Pi_{1}^{38}=\left(\frac{g_{\eta}}{q^{2}-m_{\eta}^{2}}-\frac{g_{\pi}}{q^{2}-m_{\pi}^{2}}\right)=\left(\frac{q^{2}\left(g_{\eta}-g_{\pi}\right)+\left(g_{\pi} m_{\eta}^{2}-g_{\eta} m_{\pi}^{2}\right)}{\left(q^{2}-m_{\eta}^{2}\right)\left(q^{2}-m_{\pi}^{2}\right)}\right)
$$

to facilitate comparison with Ref. [8], we find $g_{\eta}=197 \mathrm{MeV}^{2}, g_{\pi}=144 \mathrm{MeV}^{2}$, to be compared with the values $g_{\eta}=143 \mathrm{MeV}^{2}, g_{\pi}=139 \mathrm{MeV}^{2}$ extracted via the sum rule 
analysis [8]. (These values have been rescaled from those quoted in Ref. [8] by the same factor of 1.22 discussed above.) To leading order in the Borel mass, $M_{B}$ of Ref. [8],

$$
g_{\pi}=g_{\eta}=\frac{m_{u}\langle 0|\bar{u} u| 0\rangle-m_{d}\langle 0|\bar{d} d| 0\rangle}{\sqrt{3}\left(m_{\eta}^{2}-m_{\pi}^{2}\right)}
$$

which, since $\langle 0|\bar{u} u| 0\rangle=\langle 0|\bar{d} d| 0\rangle=-F^{2} B_{0}$ to leading order in the chiral expansion, implies (again to leading order) $g_{\pi}=g_{\eta}=\theta_{0} F^{2}$, the leading order chiral result. The procedure of truncating the operator product expansion (OPE) for $A_{\mu}^{3} A_{\nu}^{8}$ at operators of dimension 6 and dropping terms of $\mathcal{O}\left(m_{q}^{2}\right)$ employed in Ref. [8] thus correctly reproduces the leading order chiral constraints. The interesting $q^{2}$-dependence of $\Pi_{1}^{38}$, however, enters first only at next-to-leading order in the chiral expansion and here, comparing the results of the two approaches for the coefficient, $g_{\eta}-g_{\pi}$, of $q^{2}$ in the numerator of the last expression for $\Pi_{1}^{38}$ in Eqn. (24), ChPT to one loop predicts $g_{\eta}-g_{\pi}=53 \mathrm{MeV}^{2}$, whereas the results of Ref. [8] , rescaled for the violations of Dashen's theorem, produce $g_{\eta}-g_{\pi}=3.9 \mathrm{MeV}^{2}$, more than an order of magnitude smaller. If we consider the leading chiral behavior implicit in the sum rule result we can easily discover the source of the discrepancy. Using the expressions from Ref. [8] to evaluate $g_{\eta}-g_{\pi}$, one obtains, to $\mathcal{O}\left(m_{d}-m_{u}\right)$,

$$
g_{\eta}-g_{\pi}=\frac{\left(m_{d}-m_{u}\right)}{\sqrt{3}}\langle 0|\bar{q} q| 0\rangle\left(\frac{\left(m_{\eta}^{2}+m_{\pi}^{2}\right)}{2 M_{B}^{4}}+\mathcal{O}\left(1 / M_{B}^{6}\right)\right)
$$

where $\langle 0|\bar{q} q| 0\rangle=\langle 0|\bar{u} u| 0\rangle \simeq\langle 0|\bar{d} d| 0\rangle, M_{B}$ is the Borel mass and the higher order terms are also higher order in the meson masses (and hence higher order in the chiral expansion). Using the known chiral expansions of the meson masses, one obtains, for the leading term in the chiral expansion of $g_{\eta}-g_{\pi}$,

$$
g_{\eta}-g_{\pi}=\theta_{0} F^{2}\left(\frac{8}{9} \frac{B_{0}^{2}\left(m_{s}-\hat{m}\right)\left(m_{s}+2 \hat{m}\right)}{M_{B}^{4}}+\cdots\right)
$$

where $+\cdots$ represents terms higher order in the chiral expansion. In contrast, making the same expansion for $g_{\eta}=f_{\eta}^{2} \epsilon_{2}$ and $g_{\pi}=f_{\pi}^{2} \epsilon_{1}$ using the relevant expressions from Ref. [13], one finds

$$
g_{\eta}-g_{\pi}=\theta_{0} F^{2}\left(\frac{\left(m_{\pi}^{2}-\bar{m}_{K}^{2}\right)}{8 \pi^{2} F^{2}} \log \left(\bar{m}_{K}^{2} / \mu^{2}\right)-\frac{B_{0}\left(m_{s}-\hat{m}\right)}{8 \pi^{2} F^{2}}+\frac{32 B_{0}\left(m_{s}-\hat{m}\right)}{3 F^{2}} L_{5}^{r}+\cdots\right) .
$$


The result of Eqn. (27) begins at $\mathcal{O}\left(m_{q}^{2}\right)$, that of Eqn. $(28)$ at $\mathcal{O}\left(m_{q}\right)$ (plus the ubiquitous chiral logs): the truncation scheme of Ref. [8] has thus removed the leading contribution to the slope of $\Pi_{1}^{38}$, which accounts for the smallness of the numerical result. It would be of interest to determine which aspect of the truncation scheme is the source of the problem. The situation here appears analogous to that encountered in the sum rules analysis of the nucleon mass, where the usual approximate treatment of the continuum contributions to the phenomenological side of the sum rule leads to violations of the known chiral behavior of $M_{N}$ with $\hat{m}$, and only a more careful analysis restores the correct next-to-leading order behavior 17, 18.

In summary, we have demonstrated (1) that the mixing parameter, $\theta\left(q^{2}\right)$, of the pseudoscalar meson propagator matrix cannot be extracted, except at leading order in the chiral expansion (where it is independent of $q^{2}$ ), from a treatment of the mixed axial correlator $\Pi_{\mu \nu}^{38}$, and (2) that the QCD sum rules treatment of $\Pi_{\mu \nu}^{38}$, in the approximation of dropping terms of $\mathcal{O}\left(m_{q}^{2}\right)$ and truncating the OPE to operators of dimension 6 or less, fails completely at next-to-leading order. Since the pole approximation for $\Pi_{1}^{38}$ breaks down beyond next-toleading order, the sum rule method appears unlikely to provide any information about the $q^{2}$-dependence of $\Pi_{\mu \nu}^{38}$ beyond that already known, at next-to-leading order, from the chiral expansion, unless the multi-meson continuum is taken into account in the phenomenological side of the sum rule analysis. One could, however, profitably use the results of ChPT to provide useful constraints on the application of the sum rules method to few-body systems.

\section{ACKNOWLEDGMENTS}

The hospitality of the Department of Physics and Mathematical Physics of the Univer-

sity of Adelaide and the continuing financial support of the Natural Sciences and Research Engineering Council of Canada are gratefully acknowledged. 


\section{REFERENCES}

* Current address: Department of Physics and Mathematical Physics, University of Adelaide, Adelaide, South Australia 5005, Australia

[1] T. Goldman, J. A. Henderson and A. W. Thomas, Few-Body Systems 12 (1992) 123

[2] K. Maltman, Phys. Lett. B313 (1993) 203

[3] G. Krein, A. W. Thomas, and A. G. Williams, Phys. Lett. B317 (1993) 293

[4] J. Piekarewicz and A. G. Williams, Phys. Rev. C47 (1993) R2461

[5] J. Piekarewicz, Phys. Rev. C48 (1993) 1555

[6] K. Maltman and T. Goldman, Nucl. Phys. A572 (1994) 682

[7] T. Hatsuda, E. M. Henley, T. Meissner and G. Krein, Phys. Rev. C49 (1994) 452

[8] C. -T. Chan, E. M. Henley and T. Meissner, " $\pi-\eta$ Mixing from QCD Sum Rules", Univ. Washington preprint, 1994

[9] H. B. O'Connell, B. C. Pearce, A. W. Thomas and A. G. Williams, Phys. Lett. B336 (1994) 1

[10] K. L. Mitchell, P. C. Tandy, C. D. Roberts and R. T. Cahill, Phys. Lett. B335 (1994) 282

[11] R. Haag, Phys. Rev. 112 (1958) 669

[12] G. Ecker, "Chiral Perturbation Theory", Universitat Wien preprint UWThPh-1994-49, to appear in Progress in Particle and Nuclear Physics 35

[13] J. Gasser and H. Leutwyler, Nucl. Phys. B250 (1985) 465

[14] K. Maltman and D. Kotchan, Mod. Phys. Lett. A5 (1990) 2457

[15] J.F. Donoghue, B.R. Holstein and D. Wyler, Phys. Rev. Lett. 69 (1992) 3444; Phys. 
Rev. D47, (1993) 2089

[16] J. Bijnens, Phys. Lett. B306 (1993) 343

[17] D. K. Griegel and T. D. Cohen, Phys. Lett. B333 (1994) 27

[18] S. H. Lee, S. Choe, T. D. Cohen and D. K. Griegel, "QCD Sum Rules and Chiral Logarithms", University of Maryland preprint 95-067, HEP-PH-9411428, 1994 